\title{
THE TREATMENT OF PNEUMONIA.
}

\author{
By R. SLEIGH JOHNSON, M.D., M.R.C.P.LOND. \\ Physiciain-in-Charge of Out-patients, City of London Hospital for Diseases of the Heart and Lungs ; \\ Physician to the General Hospital, Southend-on-Sea.
}

(From Lecture given at Southend-on-Sea General Hospital.)

PNEumonia is a disease which affects all ages and stations of life. While the diagnosis as a rule affords no difficulty, the prognosis and treatment of the individual case call for the greatest skill and judgment. In the primary form of the disease, with which this lecture is in the main concerned, the infection is a self-limited one, and, barring complications, the patient will be "dead or better" in ten days. Besides natural variations in the virulence of the infecting organism and in the general resistance of the host, there are many factors which weigh the scale for or against recovery. In a disease, moreover, with a rate of spontaneous recovery as high as 70 to 80 per cent., whatever treatment be adopted, a clear judgment of the value of a particular mode of treatment is fraught with dificulty. It is easy and tempting to let enthusiasm hold the reins, and to draw pleasing but fallacious conclusions, especially from a single instance or small group of cases. Only when a large and carefully controlled series is studied can reliable information be obtained.

It is not surprising, therefore, that two opposing schools of thought have arisen On the one hand unduly optimistic claims have been made in turn for the results of treatment by a large and varying number of remedies. On the other, there is a widespread but equally unjustifiable pessimism as to the utility of any treatment at allthat the less done for a case of pneumonia the better the result. Between these two extremes lies the rational mean of relieving distressing symptoms as they arise by carefully chosen measures, with a minimum of needless disturbance and discomfort to the patient.

During the last few years increasing attention has been paid to the treatment of primary pneumonia by specific remedies. Although in suitably selected cases their value is undoubted and may be accepted as proven, general measures still remain pre-eminent in importance. Furthermore, in all cases of pneumonia, whether primary or secondary, where specific treatment is not applicable, it is solely upon such general measures that we must rely. The question of treatment may therefore be considered from three aspects :-

(I) General considerations.

(2) Non-specific therapy.

(3) Specific therapy.

(I) General Considerations.-The general considerations of treatment are too well known to need elaboration, and will therefore be briefly reviewed. Freș air, fluids, and a minimum of disturbance, are the keynotes of treatment, while adequate nursing throughout the twenty-four hours is essential. The word "pneumonia" never fails to secure for the doctor the anxious co-operation of relatives. Provided, therefore, that these facilities can be obtained at home, it is undesirable to move the patient after the third or fourth day of illness. A general word of caution may be given against 
over-treatment, especially in the late stages of the disease, by stimulant drugs; it is wiser to decide early upon a scheme of treatment and to adhere to it.

Nursing is greatly facilitated by placing the patient at the onset of illness in a single $\stackrel{\mathbb{Q}}{\complement}$ unscreened bed in the middle of the room. The temperature of the room is usually $\stackrel{.}{\Rightarrow}$ too high ; from $60^{\circ}$ to $65^{\circ} \mathrm{F}$. is sufficient. It is especially important to maintain the $\stackrel{\rho}{?}$ temperature at night in cases where superadded bronchitis is present. An open $\frac{0}{5}$ coal-fire is to be preferred to a gas-fire or an electric stove, giving better ventilation and $\frac{\overline{5}}{\overline{5}}$ less drying of the atmosphere. Windows should be open day and night. The bed- $\frac{\mathbb{\Phi}}{\Omega}$ clothes are usually too many and oppress the patient by their weight. A single blanket and an eiderdown are usually sufficient and should be raised on a cradle if necessary. $\overrightarrow{0}$ Similarly, for comfort, and to aid examination and sponging, the patient's clothing $\overrightarrow{.}$ should be light and easily removed; a pyjama jacket and trousers slit along the inner $\stackrel{\sigma}{\sigma}$ seams and secured with tapes are well suited to the purpose.

The posture adopted should be that of greatest comfort; strict recumbency is not $\frac{8}{3}$. essential, and older patients in particular may be nursed half sitting up. Any slight extra work on the heart is more than offset by the easier breathing and coughing abtained. An air-ring under the sacrum will prevent any undue local pressure.

Much benefit is derived from gentle tepid sponging every tour hours. Delirium and restlessness are lessened and sleep frequently follows; the temperature is lowered, sweating induced, and a general stimulating effect on the vasomotor centre obtained. 을 If the temperature is very high the sponging should be finished with cold water.

Every possible effort must be spared the patient. He must not be allowed to was $B$ or feed himself or attempt to reach for drinks or the sputum-pot. Nevertheless, to much disturbance and fussiness in toilet by the nurse is to be avoided. as producin unnecessary exhaustion. Visitors should be strictly limited in number and in the length of their stay.

As regards diet, seeing that the illness is a comparatively short one, the majority $\frac{\varrho}{\vec{B}}$ of pneumonia patients are over-fed. The most important consideration is to induce $\frac{0}{3}$ the patient to take as much fluid as possible to aid dilution and excretion of toxins. Success depends largely upon the ability of the nurse, whose aim it should be to give up to six pints in the twenty-four hours. Practically any bland fluid may be allowed : milk, citrated and diluted with half volume of water, imperial drink, lemonade, Horlicks' malted milk, Benger's food, bovril or chicken-broth, weak tea or coffee. The total milk in the day should not exceed two pints; two beaten-up eggs may be added. Glucose or lactose are the most readily absorbed of foods and valuable stimulants to the heart-muscle, and may be added to each feed. A pound of sugar should, if possible, be given in the day. While awake, the patient should be fed hourly, and the drinks given hot or cold as preferred.

The hygiene of the mouth and tongue is important. Dentures should be removed $N$ and the mouth cleansed with listerine or weak sodium bicarbonate solution.

Abdominal distention is usually caused by over-feeding, and may by upward $\tilde{\sigma}$ pressure seriously embarrass the heart and respiration. At the onset of illness in a $\frac{O}{D}$ vigorous patient an initial dose of $3 \mathrm{gr}$. of calomel is good treatment. Thereafter, $\stackrel{\mathbb{\infty}}{\rightarrow}$ enemata rather than purges should be relied on, a small enema saponis being given 0 every other day, or, if indicated, a turpentine or ox-gall enema. Meteorism may be relieved by a turpentine stupe or the simple passage of a rectal tube, assisted, if necessary, by a hypodermic injection of pituitrin, $\frac{1}{8}$ c.c. 
(2) Non-Specific Therapy.-The non-specific treatment of pneumonia resolves itself largely into the symptomatic relief of those factors which combine to produce exhaustion and prostration of the patient. Chief among these are pain, cough, loss of sleep, mental distress, and later, heart failure, with increasing cyanosis and dyspnœa, all evidences of the general toxæmia which, if not overcome, will result in death.

Pain due to acute pleurisy is one of the earliest features of the disease, and is often of agonizing scverity and the main cause at this stage of distress and sleeplessness. Later, with the effusion that in some degree practically always follows and the clouding of mentality which dulls appreciation, pain is less in evidence or even absent. In the early stage there should be no hesitation in giving morphia $\frac{1}{6}$ to $\frac{1}{4}$ gr., which is alone likely to relieve the pain, and repeating it if required until the third day of illness. Any depressant action on the respiratory centre is more than counterbalanced by the gain to the patient in sleep, relief of cough, and deeper, painless respirations. Later in the illness its use is not without danger. It should in any case be combined with atropine gr. $\frac{1}{10 \sigma}$ to $\frac{1}{200}$, and avoided altogether in the presence of marked cyanosis or generalized bronchitis with abundant secretion.

Other measures for the relief of pain include counter irritants to the chest, of which the lightest and most effective is antiphlogistine or kataplasma kaolin co. Burns are not infrequently seen by its application too hot to the skin. Strapping of the affected side of the chest is not recommended, as although the pain is lessened, it is at the expense of increased difficulty in respiration and in examination of the chest. Leeches are sometimes of great value, but unless skilfully applied are apt to alarm the patient. The introduction of from roo to 200 c.c. of air to produce a partial pneumothorax will also relieve the acute pain, but is not a method which can be generally applied in practice.

Cough in the early stages is frequent, painful and non-productive, preventing sleep and by the muscular effort involved exhausting the patient. A sedative linctus should therefore be given, such as Gee's linctus (tr. camph. co., oxymel scillæ, and syr. tolu aa $m \times x)$, or if a stronger drug is required codeine phosphate gr. $\frac{1}{6}$ or heroin gr. $\frac{1}{12}$, assisted by a hot drink or steam inhalation.

The routine administration of expectorants is of doubtful value and may upset digestion and appetite. Simple alkalis are free from this drawback, and may, by lessening its tenaciousness, help expectoration of the early viscous sputum. A mixture containing sodium bicarbonate or potassium citrate 3 ss with liq. ammon. acet. 3 ij will do no harm and probably also help to promote diuresis and elimination of toxins. With extensive bronchitis the addition of ammonium carbonate gr. $\mathrm{v}$ and potassium iodide gr. $\mathrm{ij}$ is worthy of trial.

Sleep is essential to recovery and must be obtained at all costs. At the onset an initial dose of morphia gr. $\frac{1}{4}$ hypodermically has no equal in reliability. When pain is less severe a combination of Dover's powder and aspirin aa gr. $x$ by mouth, or omnopon or heroin gr. $\frac{1}{6}$ hypodermically may be equally effective. In the later stages sleeplessness is due more to toxic excitement and general restlessness, and may then be relieved by various hypnotics. Chloralamide and potassium bromide gr. $\mathbf{x x}-\mathbf{x x x}$ of each may be tried, but are generally too mild. Paraldehyde $3 \mathrm{i}-\mathrm{ij}$ by mouth (or double this dose per rectum) is unpleasant but safe and usually effective, and free from depressant action on the heart. Its taste may be masked by giving it with syrup of orange, as a "sandwich" in whiskey, brandy, or lemon-juice. Of the barbitone group medinal 
gr. vij-x and allonal $\mathrm{I}$ to 2 tablets are also effective. The value of warm sponging in aiding sleep may be repeated at this stage.

Delirium may call for active restraint in addition to bed-rests. Here also sponging is often of more value than drugs. Hyoscine hydrobromide gr. $\frac{1}{10}$ may be given, but is to be regarded as a dangerous drug in that it may have the reverse effect of producing a wilder excitement than before.

The state of the heart and circulation calls for constant care and observation. Death in pneumonia occurs more often from an insidious vasomotor paralysis through toxic action on the medullary centres than from a true cardiac failure, the systolic pressure tending to fall progressively as the illness continues. The volume and tension of the pulse, the degree of cyanosis and the size of the heart are the criteria of judgment.

In the early stages, if cyanosis and venous engorgement are marked, a venesection of from half to one pint is beneficial and often more striking in its relief than drugs. Cyanosis is an indication of lack of oxygen in the blood. Its degree bears no constant relation to the extent of consolidation, which it may even precede, its early appearance being largely due to toxic damage of the alveolar epithelium preventing free interchange of gases between the blood and the alveolar air. It has been abundantly proved experimentally that the giving of oxygen will raise the oxygen-content of the arterial blood by increasing the rate of absorption from the unaffected portions of the lungs. Clinically, in addition to improvement in colour, restlessness and delirium are reduced. Oxygen therefore should be administered early at the first sign of cyanosis, and if possible maintained continuously throughout the illness. The relatives and the patien 6 should be warned that it is not a herald of impending death.

Administration through a funnel held some inches from the mouth is a wastefue and futile method and of no therapeutic value. To be of use it must be given through a rubber nasal catheter secured by strapping across the forehead or cheek or by a Bourne's nasal catheter holder. The catheter is usually tolerated if lubricated with a little 2 per cent. cocaine or novocain ointment. It should be introduced far enough to reach the posterior nares, but not so far as to cause irritation and cough by contact with the posterior pharyngeal wall. The oxygen may with advantage be warmed by bubbling through warm water, or, if desired, warm alcohol in a Woulfe's bottle. An even better method of giving oxygen, but one which is resented by some patients, is through a mask or inhaling apparatus such as that of the Apneu or Haldane design. Where the character of the breathing is very rapid and shallow, a greater depth of respiration and improved aeration of the lungs may follow the substitution of oxygen for periods by a mixture of oxygen and 5 per cent. carbon dioxide.

Turning to the consideration of drugs, the value of digitalis has been much discussed. In the opinion of the majority of clinicians it is of definite value, and should generally be given from the onset in moderate doses of $m$ xlv-lx of the tincture or its equivalent in the twenty-four hours, guided by the age and previous condition of the patient. Massive doses are not needed and may produce undesirable anorexia, vomiting and diarrhoea. Nativelle's digitalin gr. $\frac{1}{24} \sigma$ given eight-hourly is a reliable preparation more free from this risk. The early administration of digitalis is a safeguard against the otherwise serious effects of a possible auricular fibrillation later in the disease.

Even more dispute has attached to the giving of alcohol, the most used of all drugs in pneumonia. Alcohol is not a cardiac stimulant, its true action being that of a 
soporific and nervous sedative, encouraging sleep and reducing restlessness and pain, which well justifies its use in the suitable case. It also dilates the skin vessels and promotes sweating and is a readily absorbable food. Moreover, complete withdrawal of alcohol in a pneumonic patient previously accustomed to it is likely to result in delirium tremens. It is better given when indicated than as a routine, and conveniently in the form of brandy, champagne, or whiskey, in $\frac{1}{2}$ to I oz. doses up to 3 to $4 \mathrm{oz}$. in the day.

Strychnine likewise has little direct stimulant effect on the heart, but its central action on the respiratory and vasomotor centres makes it a valuable drug in the stage of falling blood pressure. It may be given hypodermically in doses of gr. $\frac{1}{64}$ in combination with atropine sulphate gr. $\frac{1}{100}$ repeated four or six-hourly when the pulse begins to flag. Other useful stimulants under like conditions are adrenalin $m \mathrm{v}-\mathrm{x}$ of the $I$ in $I, 000$ solution and pituitrin $\frac{1}{2}$ to $I$ c.c. as hypodermic injections. In acute heart failure the intravenous administration of strophanthin gr. $\frac{1}{20}$ is of value. Other direct cardiac stimulants of service are camphor gr. iij, given hypodermically in olive oil (as Curschmann's solution I c.c.) or coramine I c.c. intramuscularly four to sixhourly. Cardiazol is an organic camphor preparation with similar action. Caffeine gr. 5 to io in a mixture or given by mouth or rectum as coffee is also of help. Glucose is a valuable stimulant to the exhausted heart muscle in acute failure, and may be given intravenously as 50 c.c. of a 20 per cent. solution with 10 units of insulin subcutaneously, and repeated in twelve hours, if necessary. Larger volumes of fluid should not be given into the vein, but 250 c.c. of 5 per cent. glucose in normal saline may be given per rectum with the same object.

At the crisis a watch must be kept for signs of collapse and brandy and cardiac stimulants given should they occur. Failure of pulse and temperature to settle to normal at the expected time should arouse suspicion of a possible empyema, and lead to careful exploration with a syringe. The evidence of physical signs may be aided by the results of X-ray examination and leucocyte counts.

(3) Specific Therapy.-The whole aim of the non-specific measures of treatment described lies in keeping the patient alive until such time as his natural immunity can reach a level where the infection is overcome. Normally, circulating antibodies begin to make their appearance in the blood early on the fifth day of illness, and increase .rapidly until the seventh or eighth day when crisis occurs. It has of late been clearly shown that in a definite group of pneumonias this advent of natural immunity can be anticipated, and a high level of passive immunity temporarily conferred, by the administration early in the illness of the appropriate anti-pneumococcal serum.

Fresh interest has recently been aroused in this subject by the use in America and later in this country of the Felton concentrated anti-pneumococcal serum. It has been known for many years that the majority of cases of primary lobar pneumonia are caused by three constant and well-defined bacteriological strains of pneumococcus. Of these the relative type-incidence varies considerably from year to year and in different countries. Type 3 infection is fortunately always rare, as it has an extremely high mortality; against it no serum of protective value has been prepared. Type I infection is usually about twice as common as type 2, and both together are responsible for from half to two-thirds or more of the total cases of adult primary pneumonia. The remainder are constituted by infections due to nondescript pneumococci belonging to no classifiable bacteriological strain and known as Group 4 or non-type infections. 
Serum is of value only in Type $\mathrm{I}$ and Type 2 cases. Considering the choice of $\stackrel{\varrho}{\varrho}$ case it is usually unsuitable in old people, say over 6o years, the great majority of $\frac{\mathbb{D}}{\mathrm{D}}$ pneumococcal infections at this age being non-type. In children lobar pneumonia is $\stackrel{\varrho}{c}$ generally mild, with spontaneous recovery and absence of severe constitutional $\ddot{\vec{F}}$ disturbance, so that serum administration is unnecessary. As a rough guide its use should be restricted to adults between say 20 to 60 years, and to severe cases seen early $\frac{O}{\sigma}$ in the disease, especially during the first three days. It becomes of progressively less $\frac{\bar{m}}{\vec{*}}$ value the later it is administered, and is of no demonstrable value after the fifth day.

The largest carefully-controlled series investigated has been that published by Parke from the Bellevue, Harlem and New York hospitals in 1927-28, comprising of $\vec{o}$ Type I over $25^{\circ}$, and of Type 2 over $15^{\circ}$ serum-treated cases, and similar numbers of $\overrightarrow{\vec{\omega}}$ controls. Alternate cases were treated in order of admission, cases dying within $\stackrel{\omega}{\omega}$ twenty-four hours of admission being omitted from the series, while standards of $\overline{0}$ severity, age, physical signs and symptoms were observed and shown to be comparable $\frac{3}{6}$. in the two groups. Briefly, in Type I infection the mortality was reduced from 33 to $\dot{8}$ I9 per cent. and in Type 2 from 54 to 35 per cent. following serum administration. In Type 3 and in Group 4 cases also given serum no significant difference in mortality $\vec{F}$ was observed.

The reduction of case mortality, however, is not the sole consideration in evaluation $\vec{D}$

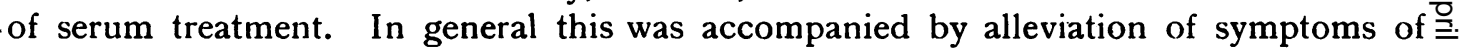
illness and curtailment of the disease, favourable results which have been confirmed in $\vec{\emptyset}$ Edinburgh and Glasgow by the Physicians to the Royal Infirmaries, and in London be Armstrong and Johnson, upon whose conclusions much of this section is based.

If laboratory facilities for sputum examination are available, unnecessary ant wasteful giving of serum may be avoided by determining before treatment the type of pneumococcal infection present. Until recently this involved a serious delay of twenty- $\frac{\bar{D}}{\square}$ four hours, using the customary method of mouse inoculation and macroscopic agglu- $\overrightarrow{\overrightarrow{0}}$ tination tests of the pneumococcus culture isolated. With the direct method of typing 3 described by Armstrong, animal inoculation can be dispensed with and type determined $\bar{F}$ directly from the sputum. In brief, a speck of the sputum to be examined is thoroughly mixed on a microscope slide with each of the three standard diagnostic sera, Types I, 23 . and 3 and coverslips applied. The specimen is then examined microscopically with the dry lens ( $\frac{1}{6}$ in. objective) and 4 eye-piece with substage condenser removed. The pneumococci, with this magnification normally just visible, in a positive reaction show a characteristic change, becoming greatly enlarged and of a ground glass appearance due $ᄋ$ to a combination of the capsular substance with the specific antibody present in the type $\frac{}{2}$ serum. The appearance is, to the practised eye, free from ambiguity. If confirmation is required it can be obtained by intra-peritoneal inoculation of a mouse with the sputum. $\widetilde{\Omega}$ In four hours or less the animal's peritonal cavity is explored with a hypodermic syringe. N The exudate so obtained, generally rich in pneumococci, when mixed with the appropriate sera shows the same characteristic change, and if organisms are numerous agglutination occurs.

Where rapid typing of the sputum cannot be carried out, treatment should be begun $\stackrel{\Phi}{\stackrel{\oplus}{\rightarrow}}$ in severe cases otherwise suitable with the combined Type $I$ and 2 serum, and continued with the appropriate single type serum, if indicated. When sputum has not been expec- $\frac{0}{D}$

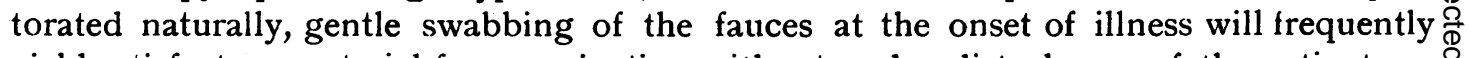
yield satisfactory material for examination without undue disturbance of the patient. 
A variety of potent sera are now available for treatment, and are alike prepared by the Felton method from the serum of immunized horses by precipitation and re-solution of the euglobulin fraction, with which the protective substances are associated. It is claimed that this process removes over 90 per cent. of the dangerous serum-protein components responsible for the production of shock and serum-sickness. Concentrates: containing a combination of approximately equal amounts of Type I and Type 2 anti. bodies, and similar concentrates effective only against Type I or Type 2 pneumococcus are supplied by reputable firms, while similar single unconcentrated sera of high potency are also available for each type of infection. Systemic disturbance is commoner after the use of unconcentrated sera, while larger volumes are necessary. It is recommended therefore that their use should be confined to cases of moderate severity.

The unit of dosage, determined by animal experiment, is standardized as that amount which will protect a mouse against ro,00o minimal lethal doses of the appropriate type pneumococcus. For therapeutic use the serum is supplied in phials containing ro,000 or 20,000 protective units each of Type I and Type 2 in a volume of 5 or Io c.c. respectively. The cost of serum still remains high and is a big factor in the general applicability of the remedy, but with larger scale production should be materially reduced. The expense is in considerable measure offset by a shortening of illness and convalescence.

It is essential that the serum should be administered intravenously at the earliest possible stage of the illness. Routine tests for protein-sensitivity are not necessary and should be reserved for cases with a known history of previous serum-administration or allergic conditions such as asthma or hay fever. In such subjects a conjunctival test is easier to perform and more reliable than an intra-dermal; two drops of $a \mathrm{I}$ in 10 dilution of the serum instilled into the conjunctival sac will in a sensitive person be followed within a quarter of an hour by reddening and lachrymation. Positive results are infrequent but if obtained call for the greatest caution; the patient can in most cases be desensitized by preliminary subcutaneous and intra-muscular injections of diluted serum at half-hour intervals in increasing amounts, beginning with o. I c.c., but unless the indications are strong it is preferable in such instances to avoid giving serum altogether.

With the use of Felton's serum anaphylactic shock is rare, and ceases to be of serious import if the simple precaution be taken never to give a dose without having available a hypodermic syringe already filled with I c.c. of $\mathrm{I}$ in I,000 adrenalin solution. Given subcutaneously without delay this will promptly relieve symptoms of shock or collapse. It is moreover found that any such disturbance becomes progressively less in degree with each subsequent injection, rarely causing anxiety after the first.

Considering the size and spacing of doses, the object of treatment is to give the largest convenient dose as early in the illness as possible without causing undue disturbance of the patient. An initial dose of 20,000 units is adequate in the majority of cases although in very severe infections this may with advantage be increased to 40,000 units. In elderly patients, or with complicating cardio-vascular disease or extensive bronchitis, it is not advisable to give more than Io,ooo units as an initial dose. The first injection should be made very slowly, taking three to five minutes, and should invariably be split, i.e. a small "pilot-dose" of $\mathrm{I}$ to 2 c.c. volume given intravenously 
followed in fifteen to twenty minutes by the main inoculum. In this way the risk of constitutional disturbance is reduced to a minimum.

The interval between doses may vary according to indications between six and twelve hours; in most instances a morning and an evening dose suffice. The total amount of serum required also varies greatly with the stage of illness and severity of the case. Briefly it may be said that for Type I cases treated early between 50,000 and 75,000 units generally suffice; for Type 2 rather more serum is needed, say, between 70,000 and 100,000 units to obtain the same effect in a comparable case. No definite rule, however, can be formulated, the clinical condition and degree of improvement being the only guides. In some early cases of moderate severity the disease appears to be arrested after one or two injections. In other more severe cases, and in those treated later in the illness, up to 150,000 units may be required before the disease is brought under control. Further, it must be stated that in some fulminating cases of excessive severity, serum appears to produce little or no beneficial effect, even when given in large doses early in the illness, the patient being prostrated and overwhelmed from the onset by the virulence of the infection.

The symptomatic effects of serum administration usually observed may be briefly summarized. In general, early treatment by Felton's serum, i.e., within the first three days, is followed by a rapid abatement of all the symptoms of illness, while in cases treated later in the disease the effects resemble more a normal subsidence by lysis. An analysis of over 100 treated and control cases, where treatment was begun not later than the fifth day, showed that the duration of fever was shortened by an average of two and a half days in Type $I$ and one and a half days in Type 2 cases.

As previously noted, true shock is rare; when present it is evidenced by tightnesse of the chest and dyspnoea of varying degree and a temporary weakening of the pulse, promptly relieved by adrenalin. Rarely, also, a rigor may follow the first or even the second injection, and be succeeded by sweating with subsequent general improvement. In early cases the temperature shows usually a critical fall to normal within twenty-four to forty-eight hours of treatment, and there remains; slight irregular fever is apt to persist for a few days in cases treated later, or if much bronchitis is present. The fall in temperature is accompanied by a fall in leucocytes and by a progressive abatement of the so-called "toxæmic" symptoms of the infection. Restlessness and mental confusion are relieved and natural sleep frequently follows. The improvement in colour and respiratory distress is often the most striking effect of treatment, even before pulse and respiration rates have subsided. Similarly, cleaning of the tongue and a return of appetite give further evidence of general improvement, accompanied often by diuresis and a disappearance of toxic albuminuria. In such successfully treated cases convalescence is materially accelerated.

In cases treated after the third and before the sixth day improvement is more gradual, the effects resembling those normally seen in subsidence by lysis. At a later stage the effects of serum are negligible and virtually impossible to assess.

The severe symptoms of serum-sickness so often seen after the giving of unconcentrated sera are not met with after the use of concentrate. Symptoms are usually absent; rarely is more disturbance seen than a transient rash or mild rise of temperature about the tenth day.

The incidence of complications appears to be unreduced by serum treatment ; on 
the other hand no evidence is forthcoming that the occurrence of pleural effusion or empyema is thereby made more likely. There is statistical evidence, however, that the treatment does lessen the liability to spread of consolidation to the opposite lung.

To summarize, Felton's serum, while of proved value, cannot like anti-diphtheritic serum be called a roo per cent. remedy, and does not benefit every case. Its use does not replace the necessity or diminish the importance of general measures of treatment, and requires throughout, care and judgment. Its exhibition in severe early cases of Type $I$ and 2 adult pneumonia is fully justified and frequently attended by the most gratifying results.

ARSENICAL JAUNDICE.

BY HUGH GORDON, M.C., M.R.c.P.

THE question of arsenical jaundice is still very much in the melting pot. This is scarcely surprising when one remembers that the ætiology of catarrhal jaundice is still unknown; likewise that of acute yellow atrophy of the liver. Similarly, the whole question of the arsenical treatment of syphilis appears to get more controversial every day. The high hopes of the sterilizatio magna of Ehrlich gave way to steady confidence in the results obtained during the Great War with the scope of treatment in bulk which it offered. But to-day we find some who say that arsenic is dangerous unless it is given in adequate doses and over an adequate period, as it tends to create an arsenic-resistant organism. The interpretation of what is adequate in both instances varies, one need hardly say, within very wide limits. Again, there are others who are gloomy of the whole future of arsenic. For instance, in Professor Dubreuilh's words : "In the treatment of syphilis the arsenical compounds are much worn out after twenty years, whereas, after four hundred years, mercury is still holding its ground."

Now the question of jaundice occurring during treatment is of relatively small moment compared with the cure of syphilis. It is, in fact, believed by some to be identical in the majority of cases with catarrhal jaundice, and can therefore be dismissed as a mere incident, having no bearing on the serious business in hand. Since, however, what are believed to be toxic cases are reported, the catarrhal school, if we may so designate them, would abstain from giving further arsenic during the continuance of the jaundice.

The school, at the other extreme, regard any case of jaundice during the administration of arsenic with extreme disquietude, fearing it to be a toxic effect of the arsenic or one of its component radicals, and would advise against any further administration of the drug, at any rate for a long period.

In between the two extremes are varying shades of opinion, which vary from placing the blame on the syphilis alone to others who blame the combination of the factor arsenic plus syphilis, or, again, to three factors, arsenic plus syphilis plus something else.

It is a problem, then, on account of the varying unknown factors quite dissimilar to arsenical dermatitis. The exact ætiology of this is also unknown, but it lends itself to fairly precise management. It can be considered as an act of God or, more medically speaking, personal idiosyncrasy. Unless premonitary signs are disregarded, it reflects no discredit on the treatment, and is more or less independent of the dose of arsenic. It has no connection with the syphilis; at least, this view is no longer seriously considered. 